\title{
Rationale for a Singapore Transthyretin Amyloidosis Registry
}

\section{Dear Editor,}

Amyloidosis is a protein mis-folding disorder, occurring when serum proteins mis-fold into unstable monomers which then self-aggregate into beta-pleated sheets of insoluble amyloid fibrils. ${ }^{1}$ The abnormal deposition of these fibrils in end-organs eventually result in organ dysfunction. Light chain (AL) amyloidosis and transthyretin (TTR) amyloidosis are the most common forms of amyloidosis seen in clinical practice. AL amyloidosis is a plasma cell dyscrasia, resulting from abnormal production of monoclonal immunoglobulin light chains by an abnormal clone of plasma cells. As such, the mainstay of treatment for AL amyloidosis is the obliteration of the plasma cell clone using chemotherapy, or stem cell transplantation.

On the other hand, TTR amyloidosis results from mis-folding of native TTR protein, which is produced in the liver. TTR is a tetrameric transporter protein produced by the liver and is ubiquitous in the blood stream. However, TTR amyloidosis occurs when these TTR tetramers dissociate and mis-fold into betapleated amyloid fibrils, which deposit in end-organs. This condition can either be secondary to an underlying genetic mutation resulting in unstable TTR tetramers, termed hereditary transthyretin amyloidosis (hATTR), or occur in the absence of apparent genetic mutation, term 'wide-type' amyloidosis (wtATTR).

TTR amyloidosis is a multi-systemic disease, with natural history and organs of involvement differing slightly between hATTR and wtATTR. wtATTR is a disease predominantly affecting elderly men, with cardiomyopathy, carpal tunnel syndrome and spinal canal stenosis as the main presenting pathologies. ${ }^{2}$

hATTR, occurring in younger patients, presents with a higher incidence of gait instability, gastrointestinal symptoms, urinary incontinence and neuropathic pain, compared to wtATTR. ${ }^{3}$ These clinical manifestations result in significant morbidity and mortality. Disease- modifying treatment, therefore, can potentially have a greater impact on this group of patients, improving their functional status, quality of life and preventing premature death. Another important aspect of hATTR is the impact on future generations. Identifying the genetic mutation in affected individuals can aid in screening or even genetic testing in first-degree relatives. While there is currently no guideline for the management of asymptomatic carriers of hATTR mutations, the knowledge gained from a disease registry can represent the first step in trying to address the many ethical challenges in managing this complex genetic disease, not only for the affected individual, but also for the family.

\section{Renewed Interests in Transthyretin Amyloidosis}

The past 5 to 10 years have truly been a renaissance period for TTR amyloidosis. Previously thought to be a benign disease affecting elderly men who died with, instead of from, the disease, we are experiencing a paradigm shift in how we understand this condition. We have gained much insight into the epidemiology of TTR amyloidosis and learnt much about how to diagnose this condition. More importantly, specific therapeutic agents have been developed for the treatment of TTR amyloidosis.

In epidemiological studies, it has been found that TTR amyloidosis is much more prevalent that we thought, and most likely under-diagnosed. In autopsy studies, $25 \%$ of individuals aged 85 and above were found to have TTR amyloid fibrils in the myocardium. ${ }^{4}$ Specific to cardiomyopathy phenotype (ATTR-CM), up to $13 \%$ of patients with the diagnosis of 'heart failure with preserved ejection fraction' (HFpEF) were found to have TTR amyloidosis as the underlying pathology. ${ }^{5}$ ATTR-CM is also increasingly being diagnosed in younger patients, with some patients having severe symptoms and even receiving heart transplantation as treatment for their heart failure. ${ }^{6}$ 
There has also been increasing awareness about the multi-systemic manifestation of TTR amyloidosis. Carpal tunnel syndrome was present in up to $68 \%$ of patients diagnosed with wtATTR, preceding the diagnosis of amyloidosis by 1 to 14 years. ${ }^{7}$ In a histological analysis of patients who underwent carpal tunnel surgery, $10 \%$ of surgical specimens were positive for amyloid deposits. ${ }^{8}$ Some of these patients were subsequently diagnosed with ATTR-CM.

There has also been a radical shift in how ATTR$\mathrm{CM}$ is being diagnosed. Previously, every patient had to undergo tissue (commonly endomyocardial) biopsy for the diagnosis of ATTR-CM. However, the current diagnostic algorithm proposes the ultilisation of technetium-based scintigraphy to replace invasive cardiac biopsy in diagnosing ATTR-CM. In patients with increased cardiac uptake on scintigraphy and absence of monoclonal proteins in the serum or urine, non-invasive testing had a $100 \%$ specificity and a $100 \%$ positive-predictive value, when compared against the gold standard of endomyocardial biopsy. ${ }^{9}$

Epidemiology and diagnostics aside, all this knowledge would have been meaningless if nothing can be done for patients after clinching the diagnosis. That would have been true 5 years ago. Various compounds for the treatment of TTR amyloidosis are now available, targeting different checkpoints of the disease process. Most of these compounds were initially studied in patients with familial amyloid polyneuropathy (FAP), showing encouraging outcomes. Studies of these novel treatment options have since been extended to patients with ATTRCM. Patisiran and inotersan inhibit TTR production at hepatocyte nucleus level. They have been shown to retard neurological deterioration and improve quality of life in FAP. ${ }^{10,11}$ Tafamidis is a stabiliser of TTR tetramers, preventing dissociation into monomers which then form the insoluble fibrils. In patients with ATTR-CM, tafamidis reduced all-cause mortality compared to placebo over 5 years, becoming the first disease modifying treatment for patients with ATTR-CM. ${ }^{12}$ Antibodies targeting serum amyloid $\mathrm{P}$ (SAP) have also shown promise in early phase studies, in promoting macrophagemediated clearance of end-organ amyloid fibrils. ${ }^{13}$ Of all the novel compounds mentioned, tafamidis has now obtained the Health Sciences Authority (HSA)'s approval for use in TTR amyloidosis, making it the first amyloidosis-specific therapy available in Singapore.

\section{Need for Data in Transthyretin Amyloidosis}

While TTR amyloidosis is not a new disease, it has suffered from years of neglect, mainly from the belief that nothing can be done for these patients. We do not have local data for the genotype, phenotype and natural history of TTR amyloidosis in the multi-ethnic Singaporean population. This is especially important, given our aging population and the impending silver tsunami facing our healthcare system.

wtATTR-CM is still predominantly seen in older patients. Diagnosing this condition in this population is notoriously difficult, given the high prevalence of hypertension, diabetes mellitus and chronic kidney disease. These conditions often affect the myocardium and mimic ATTR-CM on cardiac imaging.

From the health economics point of view, choosing the right patient population for disease-modifying treatment is also of paramount importance. Given the often-prohibitive costs of drugs in the treatment of TTR amyloidosis, financial assistance is nearly always required for patients started on these drugs. Only by knowing the natural history of this disease in our local population can we identify the patients who will derive the most benefits from receiving diseasemodifying drug treatment, as healthcare resources are limited.

Besides disease modifying drugs, organ transplantation represents the only other viable treatment option for TTR amyloidosis. In hATTR, liver transplantation can result in the cessation of the mutant TTR protein production and has been performed in patients with FAP. However, pre-transplant neurological symptoms typically remain. This treatment modality has been shown to be most beneficial in patients with the Val30Met mutation, as liver recipients with other hATTR mutations frequently show progression of cardiomyopathy despite transplantation. ${ }^{14}$ While heart transplantation has been performed in patients with advanced ATTR-CM, low organ availability in Singapore remains an issue. A local patient with advanced ATTR-CM has been implanted with a durable left ventricular assist device but that remains an expensive therapeutic option which is not readily accessible. ${ }^{15}$ With the genotype and phenotype data available in the registry, we hope to provide guidance 
in selecting patients for organ transplantation, even comparing the cost-effectiveness of transplantation versus disease-modifying drug treatment.

Lastly, from the research standpoint, the increased global interest in TTR amyloidosis provides exciting opportunities for clinicians with interest in TTR amyloidosis. Data collected from our local patients will allow us to potentially collaborate with other established amyloid registries, such as the THAOS (Transthyretin Amyloidosis Outcomes Survey) registry, to compare the characteristics of world-wide patients and our Southeast Asian cohort. ${ }^{3}$

\section{A Singapore Transthyretin Amyloidosis Registry}

Singapore's small size, well-connected healthcare systems and widespread use of healthcare informatics provide us with a unique advantage in starting a comprehensive registry, capturing patients diagnosed with this relatively uncommon condition. Although not every institution has scintigraphy and genetic testing capabilities, our island is small enough for inter-institutional collaboration, ensuring patients receive the right diagnosis and treatment no matter which part of the island they are residing in. With accurate diagnosis and data-capturing of as many patients with TTR amyloidosis into a registry, we can answer many questions about the characteristics, natural history and even genotype of our local TTR amyloidosis population. This will be a long-term multi-disciplinary endeavour, involving cardiologists, neurologists, haematologists, nuclear physicians, pathologists and geneticists with special interests in TTR amyloidosis.

Initial efforts will be focused on answering questions about the epidemiology of TTR amyloidosis in Singapore, factor contributing in delays in diagnosis, optimal diagnostic modalities/ algorithms for our local population and the impact of comorbid conditions on these patients. Subsequently, we aim to examine the different management strategies for these patients, identifying the group of patients who derive the most benefit from expensive disease-modifying treatment. Lastly, we hope to study the long-term natural history of our local TTR amyloidosis patients - those who receive disease-modifying treatment as well as in those who do not.

\section{Conclusion}

The field of amyloidosis has been given a new lease of life over the past 5 years, especially transthyretin amyloidosis. New non-invasive algorithms have been developed for diagnosing this condition and, for the first time, evidence-based treatment with proven benefits can be offered to TTR amyloidosis patients. The approval of the first disease-modifying drug by the HSA is truly a promising start, as we await other treatment agents to complete their trial and potentially enter the local scene. A national effort will be definitely important for us to better understand the characteristics of our local TTR amyloidosis patients, potentially developing a local screening and diagnostic algorithm best suited to our unique healthcare infrastructure. Lastly, it provides us a platform to hop onto the global research bandwagon, with the eventual aim of establishing Singapore as a regional centre of excellence for TTR amyloidosis treatment and research.

\section{REFERENCES}

1. Ruberg FL, Grogan M, Hanna M, Kelly JW, Maurer MS. Transthyretin Amyloid Cardiomyopathy: JACC State-of-the-Art Review. J Am Coll Cardiol. 2019;73:2872-91.

2. Nativi-Nicolau J, Maurer MS. Amyloidosis cardiomyopathy: update in the diagnosis and treatment of the most common types. Curr Opin Cardiol. 2018;33:571-9.

3. Damy T, Kristen AV, Suhr OB, Maurer MS, Planté-Bordeneuve V, $\mathrm{Yu}$ CR, et al. Transthyretin cardiac amyloidosis in continental Western Europe: an insight through the Transthyretin Amyloidosis Outcomes Survey (THAOS). Eur Heart J. 2019. pii: ehz173.

4. Maurer MS, Elliott P, Comenzo R, Semigran M, Rapezzi C. Addressing Common Questions Encountered in the Diagnosis and Management of Cardiac Amyloidosis. Circulation. 2017;135:1357-77.

5. González-López E, Gallego-Delgado M, Guzzo-Merello G, de Haro-Del Moral FJ, Cobo-Marcos M, Robles C, et al. Wild-type transthyretin amyloidosis as a cause of heart failure with preserved ejection fraction. Eur Heart J. 2015;36:2585-94.

6. Rosenbaum AN, AbouEzzeddine OF, Grogan M, Dispenzieri A, Kushwaha S, Clavell A, et al. Outcomes After Cardiac Transplant for Wild Type Transthyretin Amyloidosis. Transplantation. 2018;102:1909-13.

7. Nakagawa M, Sekijima Y, Yazaki M, Tojo K, Yoshinaga T, Doden T, et al. Carpal tunnel syndrome: a common initial symptom of systemic wild-type ATTR (ATTRwt) amyloidosis. Amyloid. 2016;23:58-63.

8. Sperry BW, Reyes BA, Ikram A, Donnelly JP, Phelan D, Jaber WA, et al. Tenosynovial and Cardiac Amyloidosis in 
Patients Undergoing Carpal Tunnel Release. J Am Coll Cardiol. 2018;72:2040-50.

9. Gillmore JD, Maurer MS, Falk RH, Merlini G, Damy T, Dispenzieri A, et al. Nonbiopsy Diagnosis of Cardiac Transthyretin Amyloidosis. Circulation. 2016;133:2404-12.

10. Adams D, Gonzalez-Duarte A, O'Riordan WD, Yang CC, Ueda M, Kristen AV, et al. Patisiran, an RNAi Therapeutic, for Hereditary Transthyretin Amyloidosis. N Engl J Med. 2018;379:11-21.

11. Benson MD, Waddington-Cruz M, Berk JL, Polydefkis M, Dyck PJ, Wang AK, et al. Inotersen Treatment for Patients with Hereditary Transthyretin Amyloidosis. N Engl J Med. 2018;379:22-31.

12. Maurer MS, Schwartz JH, Gundapaneni B, Elliott PM, Merlini G, Waddington-Cruz M, et al. Tafamidis Treatment for Patients with Transthyretin Amyloid Cardiomyopathy. N Engl J Med. 2018;379:1007-16.

13. Richards DB, Cookson LM, Berges AC, Barton SV, Lane T, Ritter JM, et al. Therapeutic Clearance of Amyloid by Antibodies to Serum Amyloid P Component. N Engl J Med. 2015;373:1106-14.
14. Benson MD. Liver transplantation and transthyretin amyloidosis. Muscle Nerve. 2013;47:157-62.

15. Lim CP, Lim YP, Lim CH, Ong HY, Tan D, Chew HC, et al. Ventricular Assist Device Support in End-Stage Heart Failure From Cardiac Amyloidosis. Ann Acad Med Singapore. 2019; $48: 435-438$.

Weiqin Lin, ${ }^{1,2}$ MBBS, MRCP, MMed (Int Med)

${ }^{1}$ Department of Cardiology, National University Heart Centre, Singapore. Singapore

${ }^{2}$ Yong Loo Lin School of Medicine, National University of Singapore. Singapore

Address for Correspondence: Dr Lin Weiqin. Department of Cardiology, National University Heart Centre, Singapore; 1E Kent Ridge Road, NUHS Tower Block, Level 9, Singapore 119228

Email: weiqin_lin@nuhs.edu.sg 